\title{
The nuclear starburst activity in the Seyfert 2 galaxy NGC 7679
}

\author{
Q. S. Gu${ }^{1}$, J. H. Huang ${ }^{1}$, J. A. de Diego ${ }^{2}$, D. Dultzin-Hacyan ${ }^{2}$, S. J. Lei ${ }^{1}$, and E. Benítez ${ }^{2}$ \\ 1 Department of Astronomy, Nanjing University, Nanjing 210093, PR China \\ 2 Instituto de Astronomía, Universidad Nacional Autónoma de México, Apdo Postal 70-264, Mexico D.F. 04510, \\ México
}

Received 28 May 2001 / Accepted 5 June 2001

\begin{abstract}
We present our recent spectrophotometric results of the infrared luminous Seyfert 2 galaxy NGC 7679. We find compelling evidence for the presence of a recent nuclear starburst, revealed by the observations of a) the spectral features of high order Balmer absorption lines, b) the weak equivalent widths for CaIIK $\lambda 3933$, CN $\lambda 4200, G$-band $\lambda 4300$ and MgIb $\lambda 5173$, and c) the suggested UV stellar wind resonance lines (N V $\lambda 1240$, Si IV $\lambda 1400$ and C IV $\lambda 1550$ ) in the IUE spectrum. Using the simple stellar population synthesis model, we find that in the nuclear 2" $\times 2$ " region, the contributions from the old, intermediate and young components are $21.7 \%, 42.9 \%$ and $35.4 \%$, respectively. These nuclear starburst activities might have been triggered by the close encounter with NGC 7682, as suggested by recent numerical simulations.
\end{abstract}

Key words. galaxies: active - galaxies: Seyfert - galaxies: starburst - galaxies: stellar content

\section{Introduction}

Observational evidence supporting that a nuclear/circumnuclear starburst coexists with the central active nucleus has increased recently. In the study of a sample of Seyfert 2 galaxies, Storchi-Bergmann et al. (2000) and Gonzalez Delgado et al. (2001) have found that about 30 to $50 \%$ of Seyfert 2 galaxies have nuclear starbursts. Since both of them might be related to gas inflow, which could be triggered by an axis-asymmetric perturbation (such as bars, tidal interactions or mergers), there is extensive speculation on the connection between starburst and nuclear activity (Norman \& Scoville 1988; Terlevich et al. 1990; Heckman et al. 1995; Heckman et al. 1997; Gonzalez Delgado 1998; and see the recent review by Veilleux 2000). Gu et al. (2001) studied 51 Seyfert 2 galaxies from data available in the literature, and found that Seyfert 2 galaxies with no evidence of a hidden Seyfert 1 nucleus have characteristics in common with starburst galaxies.

There are several observable spectral signatures that reveal the nuclear starburst in Seyfert 2 galaxies. For example, in the ultraviolet (UV) band one can use the stellar wind resonance lines, such as N V $\lambda 1240$, Si IV $\lambda 1400$ and C IV $\lambda 1550$ (Heckman et al. 1995; Heckman et al. 1997; Gonzalez Delgado et al. 1998). In the optical, the broad emission lines of Wolf-Rayet stars around $4686 \AA$ (Kunth \& Contini 1999) and high order Balmer ab-

Send offprint requests to: Q. S. Gu,

e-mail: qsgu@nju.edu.cn sorption lines originating in the photosphere of $\mathrm{O}, \mathrm{B}$ and $\mathrm{A}$ stars (Gonzalez Delgado et al. 1999; Gonzalez Delgado et al. 2000) are indicators. Recently, Cid Fernandes et al. (2001) have suggested a series of empirical criteria for the existence of a starburst in Seyfert 2 galaxies, such as weak CaIIK $\lambda 3933$, low excitation lines and large far-infrared luminosity, etc.

In this paper, we report the presence of spectral signatures of nuclear starbursts in the infrared luminous Seyfert 2 galaxy NGC 7679, with the infrared luminosity, $L_{\mathrm{IR}}$, of $1.1 \times 10^{11} L_{\odot}$ (Veilleux et al. 1995; Veron-Cetty \& Veron 2000) and the redshift $(z) 0.01714$ (corresponding to a distance of $68.5 \mathrm{Mpc}$, for $H_{0}=75 \mathrm{~km} \mathrm{~s}^{-1} \mathrm{Mpc}^{-1}$ and $q_{0}=0$ ). NGC 7679 is one member of Arp 216, the other is NGC 7682 , their projected separation is $89.6 \mathrm{kpc}$ (Arp 1966). We suggest that the starburst activity in this galaxy might be related to the tidal interaction with NGC 7682, as indicated by recent numerical simulations (Barnes \& Hernquist 1996; Mihos \& Hernquist 1996). This paper is organized as follows. In Sect. 2 we describe our observations, data reduction and results. In Sect. 3 we discuss our results and we summarize our conclusions in Sect. 4 .

\section{Observations and results}

Spectra of NGC 7679 were obtained on Sep. 28-29, 2000, with the Boller \& Chivens spectrograph attached to the $2.1 \mathrm{~m}$ telescope of Observatorio Astronómico Nacional 


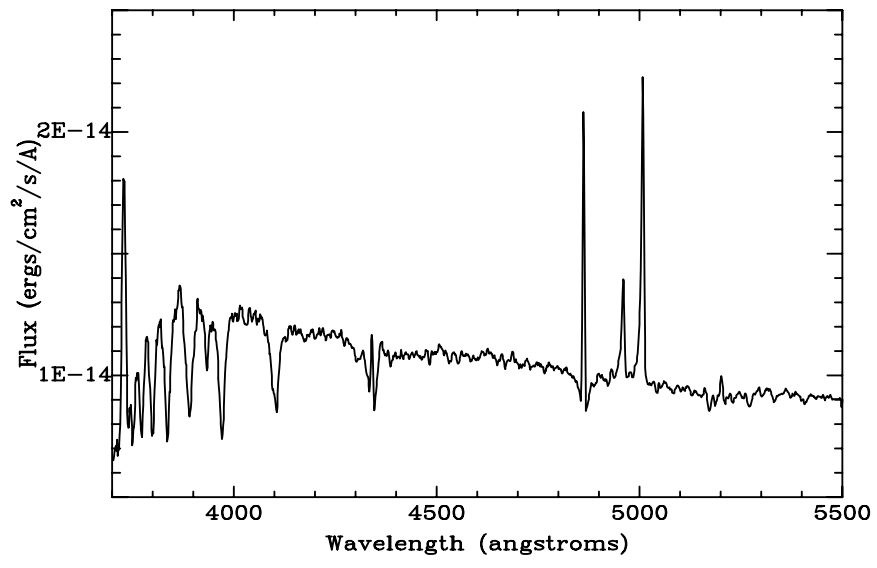

Fig. 1. The spectrum of NGC 7679, where we could see the clear higher-order Balmer absorption lines.

at San Pedro Martir (Mexico). We used a Thomson $2048 \times$ $2048 \mathrm{CCD}$ and a 600 lines/mm grating, which provides a dispersion of $83 \AA / \mathrm{mm}$. The width of the long slit was set to $2^{\prime \prime}$ (i.e., 664 pc for NGC 7679). HeAr-spectra had been taken before and after the object spectra for wavelength calibration, and BD +284211 was selected from KPNO standards for flux calibration. The total exposure time was $10800 \mathrm{~s}(2700 \mathrm{~s} \times 4)$. All spectra were reduced using standard IRAF ${ }^{1}$ procedures.

In Fig. 1, we show the central $2^{\prime \prime} \times 2^{\prime \prime}$ region spectrum of NGC 7679, and in Table 1, we list all emission and absorption lines that we identify in the spectrum along with their respective equivalent widths $(E W \mathrm{~s})$. Stellar absorption wings clearly evolve the $\mathrm{H} \beta$ and $\mathrm{H} \gamma$ emission lines. For these lines, fluxes and $E W$ s were obtained using the IRAF-specfit routine. The spectrum also shows the presence of high-order Balmer absorption lines (up to H12), and several weak absorption lines, such as, CaIIK $\lambda 3933$, CN $\lambda 4200, G$-band $\lambda 4300$ and MgIb $\lambda 5173$.

\section{Discussion}

\subsection{Stellar populations}

To estimate the stellar population in the nuclear region of NGC 7679, we compare our observed spectrum with the evolutionary synthesis model, GISSEL96 (Bruzual \& Charlot 1996). Following Cid Fernandes et al. (2001), we use three components: an old and an intermediate population, with ages $>1$ Gyr and $\sim 10^{8}$ yr, respectively, and an $f_{\lambda} \sim \lambda^{-1}$ power-law component to account for either a scattered AGN continuum or a young starburst component, since it is impossible to disentangle the power-law AGN continuum from the contribution of a young starburst of an age $\leq 10$ Myr (Storchi-Bergmann et al. 2000; Gonzalez Delgado et al. 2001; Cid Fernandes et al. 2001).

\footnotetext{
${ }^{1}$ IRAF is distributed by the National Optical Astronomy Observatory, which is operated by the Association of Universities for Research in Astronomy, Inc., under cooperative agreement with the National Science Foundation.
}

Table 1. Absorption and emission lines in NGC 7679.

\begin{tabular}{llc}
\hline \hline Wavelength $(\AA)$ & Ion & Equivalent Width $(\AA)$ \\
\hline 3727 & {$[\mathrm{O} \mathrm{II}]$} & $16.33^{e}$ \\
3750 & $\mathrm{H} 12$ & 2.47 \\
3770 & $\mathrm{H} 11$ & 4.36 \\
3797 & $\mathrm{H} 10$ & 5.32 \\
3835 & $\mathrm{H} 9$ & 6.62 \\
3889 & $\mathrm{H} \zeta$ & 7.25 \\
3933 & $\mathrm{CaII} \mathrm{K}$ & 1.41 \\
3970 & $\mathrm{H} \epsilon$ & 6.73 \\
4101 & $\mathrm{H} \delta$ & 6.79 \\
4200 & $\mathrm{CN}$ band & 0.35 \\
4300 & $\mathrm{G}$ band & 0.68 \\
4340 & $\mathrm{H} \gamma$ & $2.92^{a, e}$ \\
4861 & $\mathrm{H} \beta$ & $9.72^{b, e}$ \\
4959 & {$[\mathrm{O}$ III $]$} & $3.42^{e}$ \\
5007 & {$[\mathrm{O}$ III $]$} & $9.74^{e}$ \\
5173 & $\mathrm{MgIb}$ & 0.82 \\
\hline
\end{tabular}

${ }^{a}$ Corrected for stellar absorption of 6.0 A using specfit.

${ }^{b}$ Corrected for absorption of $5.7 \AA$.

${ }^{e}$ Means for emission lines.

We normalized all spectra at $4800 \AA$, and we compared parameters of continuum fluxes at 4020, 4510 and $5500 \AA$, as well as $E W$ s of the absorption lines for CaIIK $\lambda 3933$, CN $\lambda 4200$ and $G$-band $\lambda 4300$. The relative errors on these quantities are set to less than $5 \%$. Figure 2 shows the observed spectrum and the three component population synthesis fit. The fractions of the power-law component, the intermediate and the old populations contributing to the total $4800 \AA$ monochromatic light are $35.4 \%, 42.9 \%$ and $21.7 \%$, respectively.

For comparison, we make use of the empirical formulae by Cid Fernandes et al. (2001) to derive the fractions of old and young population, the results are for the young, intermediate and old population, the fractions are $33.5 \%$, $48.3 \%$ and $18.2 \%$, respectively, which are consistent with our fitting results.

\subsection{UV spectroscopy}

The presence of high-order Balmer absorption lines indicate clearly the existence of intermediate-age stars in the nuclear region of NGC 7679. But the origin of the power-law component, accounting for about $35 \%$ of the total $4800 \AA$ flux, may be the result of a young starburst or a scattered AGN continuum.

It is well known that the stellar wind resonance absorption lines (N V $\lambda 1240$, Si IV $\lambda 1400$ and C IV $\lambda 1550$ ) are unambiguous signatures of young starbursts in the $U V$ band, 


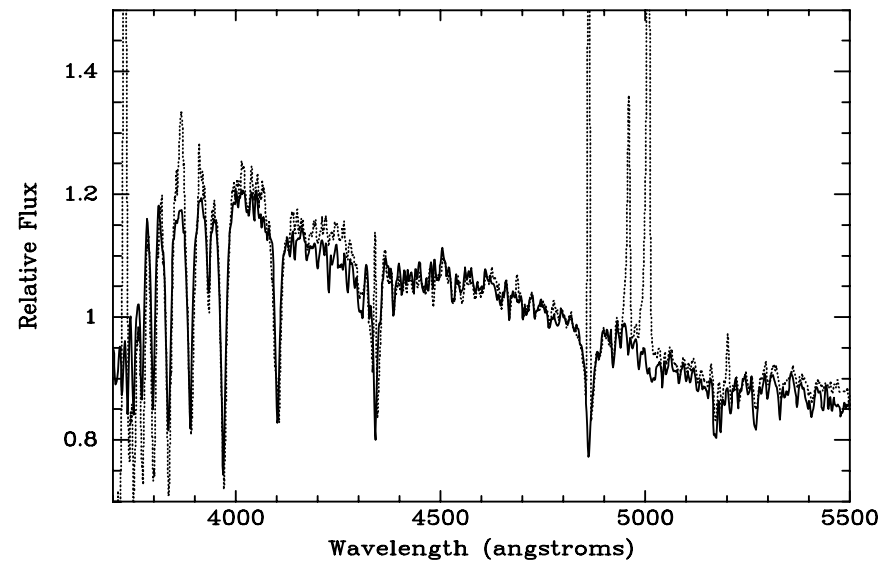

Fig. 2. The best-fitting spectrum for NGC 7679 with the threecomponent population synthesis model (solid line), which is obtained by combining a power-law component with an intermediate age $\left(10^{8} \mathrm{yr}\right)$ and an old $(>1 \mathrm{Gyr})$ population. The relative contributions to the total $4800 \AA$ monochromatic light are $35.4 \%, 42.9 \%$ and $21.7 \%$, respectively. For comparison, the observed spectrum is superimposed (dotted line).

as suggested by Heckman et al. (1995), Heckman et al. (1997), and Gonzalez Delgado et al. (1998). Unfortunately, these features can only be observed clearly in the brightest $U V$ galaxies (Heckman et al. 1997; Gonzalez Delgado et al. 1998).

Is there any indication of a young starburst in the $U V$ spectrum of NGC 7679 ? We retrieved the $U V$ spectrum for this object from the IUE public archive (Fig. 3). For comparison, we also plot the IUE and HST GHRS spectra of NGC 5135. NGC 5135 is one of the Seyfert 2 galaxies that presents clear nuclear starburst activity and high order Balmer absorption lines (Gonzalez Delgado et al. 1998).

Comparing IUE with HST GHRS spectra of NGC 5135, we checked that all typical absorption lines (as marked in Fig. 3) in high resolution and high $S / N$ GHRS spectrum are also present in the IUE data, although, the $S / N$ is low. At the same time, it is clear that all these stellar wind absorption lines are also present in NGC 7679; in particular, we could see the clear PCygni profile of C IV $\lambda 1550$, which is even more significant than that in NGC 5135. Although the IUE spectrum is marginal, we find the clear signature of a young starburst in the nuclear region of NGC 7679.

That the power-law component is the contribution of a young starburst found in this work for NGC 7679 is consistent with a recent work by Cid Fernandes et al. (2001). These authors have found that all $x_{\mathrm{FC}} \geq 30 \%$ sources are confirmed Seyfert 2/starburst composites (where $x_{\mathrm{FC}}$ means the contribution from a power-law featureless continuum) and in these sources, the featureless continuum is dominated by a nuclear/circumnuclear starburst, because in Seyfert 2 galaxies the scattered light from the central AGN cannot exceed $\sim 30 \%$, otherwise we would observe the reflected broad lines directly and the galaxy would no longer be classified as a Seyfert 2 galaxy.

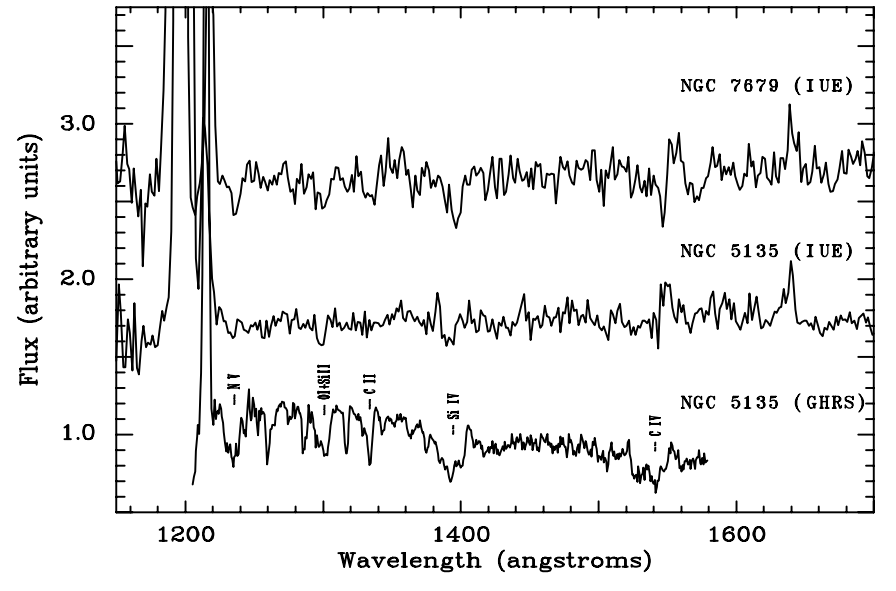

Fig. 3. Comparison the IUE UV spectrum of NGC 7679 with NGC 5135, and IUE with HST GHRS for NGC 5135. Although the IUE spectrum of NGC 5135 has a somewhat low $S / N$, all these marginal absorption lines are clearly present in the GHRS spectrum. Several stellar wind absorption lines, such as N V $\lambda$ 1240, Si IV $\lambda 1400$ and C IV $\lambda$ 1550, can also be identified in the IUE spectrum of NGC 7679 .

\section{Conclusions}

In this paper, we present unambiguous evidence for the recent starburst activity in the nuclear region of NGC 7679: the higher-order Balmer absorption lines and weaker CaIIK $\lambda 3933$, CN $\lambda 4200, G$-band $\lambda 4300$ and MgIb $\lambda 5173$; and in the $U V$ band, several stellar wind absorption lines. Using the simple three-component stellar population synthesis model, we obtain that the nuclear stellar population could be $42.9 \%$ intermediate age $\left(10^{8} \mathrm{yr}\right), 21.7 \%$ old ( $>1 \mathrm{Gyr}$ ), combined with a $35.4 \%$ power-law component, which might be from the young massive nuclear starburst activity.

Acknowledgements. We would like to thank Dr. Rosa Gonzalez Delgado for sending us her HST GHRS spectra. Q. S. G. acknowledges support from UNAM postdoctoral program (Mexico) and support from National Natural Science Foundation of China and the National Major Project for Basic Research of the State Scientific Commission of China. And D. D.-H., J. A. D. and E. B. acknowledge support from grant IN 115599 from PAPIIT-UNAM. This research has made use of NASA's Astrophysics Data System Abstract Service and the NASA/IPAC Extragalactic Database (NED) which is operated by the Jet Propulsion Laboratory, California Institute of Technology, under contract with the National Aeronautics and Space Administration.

\section{References}

Arp, H. C. 1966, ApJS, 14, 1

Barnes, J. E., \& Hernquist, L. 1996, ApJ, 471, 115

Bruzual, A. G., \& Charlot, S. 1996, Galaxy Isochrone Syntheis Spectral Evolution Library, AAS, CD-ROM Series, vol. VII (GISSEL 96) 
Cid Fernandes, R., Heckman, T., Schmitt, H., Gonzalez Delgado, R. M., \& Storchi Bergmann, T. 2001, ApJ, in press [astro-ph/0104186]

Gonzalez, Delgado, R. M., Heckman, T., Leitherer, C., et al. 1998, ApJ, 505, 174

Gonzalez Delgado, R. M., Leitherer, C., \& Heckman, T. 1999, ApJS, 125, 489

Gonzalez Delgado, R. M., Heckman, T., \& Leitherer, C. 2000 [astro-ph/0001104]

Gonzalez Delgado, R. M., Heckman, T., \& Leitherer, C. 2001, ApJ, 546, 845

Gu, Q. S., Dultzin-Hacyan, D., \& de Diego, J. A. 2001, Rev. Mex. Astron. Astrofis., 37, 3

Heckman, T. M., Krolik, J., Meurer, G., et al. 1995, ApJ, 452, 549
Heckman, T. M., Gonzalez-Delgado, R. M., Leitherer, C., et al. 1997, ApJ, 482, 114

Kunth, D., Contini, T. 1999, in IAU Symp. 193, Wolf-Rayet Phenomena in Massive starts and Starburst Galaxies, ed. K. A. van der Hucht, G. Koenigsberger, \& P. R. J. Eenens (San Francisco: ASP), 725

Mihos, J. C., \& Hernquist, L. 1996, ApJ, 464, 641

Norman, C., \& Scoville, N. 1988, ApJ, 332, 124

Storchi-Bergmann, T., Raimann, D., Bica, E. L. D., \& Fraquelli, H. A. 2000, ApJ, 544, 747

Terlevich, E., Diaz, A. I., \& Terlevich, R. 1990, MNRAS, 242, 271

Veilleux, S., Kim, D. C., Sanders, D. B., Mazzarella, J. M., \& Soifer, B. T. 1995, ApJS, 98, 171

Veilleux, S. 2000 [astro-ph/0012121]

Veron-Cetty, M. P., \& Veron, P. 2000, ESO Sci. Rep., 19, 1 